\title{
Gender differences in folate status are associated with cognition in healthy Irish elderly adults
}

\author{
E. M. O'Connor ${ }^{1}$, G. F. Fitzgerald ${ }^{1,2}$ and P. W. O'Toole ${ }^{1,2}$ on behalf of the eldermet consortium \\ (see http://eldermet.ucc.ie for consortium membership) \\ ${ }^{1}$ Department of Microbiology and ${ }^{2}$ Alimentary Pharmabiotic Centre, University College, Cork, Ireland
}

In elderly populations, cognitive impairment and incident dementia may be related to inadequate vitamin B status, predominantly folate and vitamin $\mathrm{B}_{12}$. Folate deficiency has been associated with Alzheimer's disease as well as preceding the onset of other dementia ${ }^{(1)}$, while low folate and vitamin $B_{12}$ have been associated with depressive symptoms ${ }^{(2)}$. Functional deterioration may also increase with folate deficiency $^{(3)}$. The aim of this study was to examine associations between B vitamins (serum folate and vitamin $\mathrm{B}_{12}$ ) and measures of cognition in a sample of healthy Irish adults.

Serum vitamin $\mathrm{B}_{12}$ and folate concentrations were measured by ELISA in 194 community-dwelling and day hospital patients (aged 64-95 years) who participated in the ELDERMET project. Cognitive and functional evaluation included the Mini Mental State Exam (MMSE) and Barthel Index of Activities of Daily Living, while the Geriatric Depression scale was used as a screening tool for depression.

\begin{tabular}{|c|c|c|c|c|c|c|c|}
\hline & \multicolumn{3}{|c|}{ Male $(n=84)$} & \multicolumn{3}{|c|}{ Female $(n=105)$} & \multirow[b]{2}{*}{$P$ value $^{1}$} \\
\hline & Mean & Median & SD & Mean & Median & SD & \\
\hline Age & 75.6 & 75.0 & 7.0 & 75.1 & 75.0 & 6.8 & 0.689 \\
\hline BMI & 28.4 & 28.2 & 4.0 & 26.9 & 25.8 & 6.2 & 0.01 \\
\hline Serum folate $(\mathrm{ng} / \mathrm{ml})$ & 7.1 & 6.3 & 3.6 & 9.1 & 7.0 & 5.0 & 0.012 \\
\hline Serum vitamin B12 (ng/l) & 244.0 & 224.0 & 104.4 & 285.1 & 260.0 & 117.1 & 0.014 \\
\hline Mini Mental State Exam ${ }^{2}$ & 27.7 & 29.0 & 2.5 & 27.6 & 28.0 & 2.6 & 0.606 \\
\hline Barthel Index ${ }^{3}$ & 19.7 & 20.0 & 1.7 & 19.2 & 20.0 & 1.7 & 0.407 \\
\hline Geriatric Depression test ${ }^{4}$ & 2.0 & 1.0 & 2.1 & 2.2 & 1.0 & 2.3 & 0.694 \\
\hline Charlson Index of co-morbidity ${ }^{5}$ & 2.4 & 2.0 & 1.4 & 1.9 & 2.0 & 1.3 & 0.05 \\
\hline
\end{tabular}

Linear regression analysis showed that folate and vitamin $\mathrm{B}_{12}$ were positively associated (adj. $R^{2}$ for model $\left.=0.14, \beta=0.387, P=0.001\right)$. Male subjects had significantly lower mean folate $(P=0.012)$ and vitamin $\mathrm{B}_{12}(P=0.014)$ status than females (see Table). After stratification by gender, linear regression analyses in females showed folate status to be positively associated with MMSE score (adj. $R^{2}$ for model $=0.10, \beta=0.333, P=0.003$ ) accounting for age and BMI. These associations were not evident in males. A trend for increased comorbidity was also observed in male subjects compared to females $(P$ for trend $=0.055)$. Folate and vitamin $\mathrm{B}_{12}$ status were not associated with other measures of cognition, functionality and depression in males or females.

A one-way ANOVA showed a significant increase (improvement) in MMSE scores with increasing tertile of folate status in females $(P=0.004)$. Subjects in the lowest tertile of folate had a significantly lower MMSE score (mean score $=26.0)$ compared to those in the middle (mean score $=27.9)$ and highest tertiles (mean score $=28.4)$. These associations were not present in males $(P>0.1)$.

In conclusion, folate status was better in females compared to males. Cognitive function, measured by MMSE score, increased with increasing folate status in females but not in males. Folate status in the elderly should be monitored as inadequacy could contribute to the brain ageing process and cognitive decline.

1. Tettamanti et al. (2006) J Am Coll Nutr 25, 502-508.

2. Robinson et al. (2011) Int J Geriatr Psychiatry 26, 307-313.

3. Johnson et al. (1990) Vitamin Disorders in the Elderly. pp. 117-147. New York: Raven Press Ltd. 